\title{
TRANSPORT IN AN ERGODIC MAGNETIC FIELD WITH AMBIPOLAR ELECTRIC FIELD EFFECTS
}

\author{
$\underline{\text { W. Feneberg }}^{*}$ and M. A. Hellberg ${ }^{\dagger}$ \\ "Max-Planck-Institut für Plasmaphysik, \\ EURATOM Association, D-8046 Garching, Fed. Rep. of Germany \\ $\dagger$ Plasma Physics Research Institute, \\ University of Natal, Durban, South Africa
}

\section{INTRODUCTION}

Stochastic magnetic fields may be applied to tokamak edges to establish an ergodic divertor [1] or they may arise spontaneously due to instabilities. In addition, they may play an important role in the $\mathrm{H}$-mode, since Neuhauser, et al [2] have recently shown that asymmetries in the deposition profiles on ASDEX divertor plates may be explained by imperfectly installed multipole windings leading to a stochastic magnetic layer just inside the separatrix.

Transport in a stochastic magnetic field has been investigated in a number of earlier papers based on the quasilinear theory $[3,4]$, resulting in the well known diffusion coefficient of Rechester and Rosenbluth [3] for the case of a steady-state perturbation field. Thyagaraja, et al [5], using a simplified Fokker-Planck equation, considered a fluctuating stochastic magnetic field and found a large increase of the transport in the long mean free path regime.

In this paper we use the drift kinetic equation, including the effect of the electric field, together with the Krook (or BGK) collision model [6], which conserves particles, momentum and energy. We consider a fluctuating stochastic magnetic field. It should be noted that even for a time-independent applied perturbation, a frequency arises through the azimuthal electron drift $V_{E}=-(\nabla \phi \times \vec{B}) / B^{2}$.

In agreement with previous theory we find that only modes that are in resonance with the unperturbed magnetic surfaces contribute to the transport, but with a coefficient that is larger by a factor $\gamma=2 \lambda_{\epsilon} / R q$ than that derived from quasilinear theory ( here $\lambda_{e}$ is the electron mean free path, $R$ the torus major radius and $q$ the safety factor). In particular we find that inclusion of momentum conservation in like-particle collisions plays a significant role. Using a simpler collision model that conserves only particle number, we also obtain an estimate for the width of the resonant layer. We find that transport occurs in the range

$$
0 \leq|(m-n q)| \leq\left(\frac{\lambda_{e}}{r q}\right)^{-1}\left(\frac{\omega}{\nu}\right)^{1 / 2}
$$

Here $m$ and $n$ are the poloidal and toroidal winding numbers, and $\omega$ and $\nu$ the fluctuation and collision frequency, respectively. 
We also present a self-consistent solution for the radial electric field based on a model in which the radial electron transport is anomalous due to motion along the stochastic field lines and the ion transport is neoclassical. Although there is no detailed flux balance, overall ambipolarity yields a relation between the radial electric field and the poloidal rotation velocity. Radial ion momentum balance and toroidal momentum balance of the whole fluid enables one then to solve for the electric field, as well as the poloidal and toroidal rotation velocities. It is found that the neoclassical perpendicular ion current produces a toroidal rotation in the Co-direction, of approximately $V_{i, \phi}=-P^{\prime} / e n_{e} B_{\theta}$, where $P^{\prime}$ is the total radial pressure gradient and $B_{\theta}$ the poloidal magnetic field.

\section{COLLISIONAL TRANSPORT EQUATIONS}

We consider cylindrical flux surfaces perturbed by a weak stochastic magnetic field with radial component $b_{r}$, which we expand as

$$
b_{r}=\sum \sum \sum b_{\omega, m, n}(r) \exp (i(\omega t+m \theta-n \phi)) .
$$

The particle flux perpendicular to the unperturbed flux surfaces is given by

$$
\Gamma_{r}=<b_{r} \int f v_{\|} d^{3} v>,
$$

where $\langle.$.$\rangle represents a time-average over the magnetic surfaces, i.e. over \theta, \phi$ and $t$, and $f$ is the total distribution function.

To find $f$ we solve the drift kinetic equation for the electrons in the rotating system, given by $V_{E}$. We use the cylindrical approximation, neglecting the $\vec{B} \times \nabla B$ drift,

$$
\frac{\partial f}{\partial t}+v_{\|} \hat{B} \cdot \nabla f-\frac{q}{m}(\hat{B} \cdot \nabla \phi) \frac{\partial f}{\partial v_{\|}}=\nu_{e, e}\left(f_{e, e}^{M}-f\right)+\nu_{e, i}\left(f_{e, i}^{M}-f\right) .
$$

The Maxwellian $f_{e, e}^{M}$ has the self-consistent values of density $n_{e}$, drift $\overrightarrow{u_{e}}$ and temperature $T_{e}$ of the total (perturbed) distribution, i.e.

$$
f_{e, e}^{M}=n_{e}\left(\frac{m_{e}}{2 \pi T_{e}}\right)^{\frac{3}{2}} \exp \left[-\left\{\frac{m_{e}}{2 T_{e}}\left(\left(v_{\|}-u_{\|}\right)^{2}+2 \mu B\right)\right\}\right] .
$$

Here $\mu$ is the magnetic moment. We see that particle number, momentum and energy are conserved in like-particle collisions [6]. For electron-ion collisions we conserve particles and energy, but momentum transfer is allowed, representing electron-ion friction. The distribution function is expanded, $f=f_{0}+f_{1}$, with $f_{0}$ a Maxwellian in the unperturbed magnetic field and $f_{1}$ being represented by a series similar to that for $b_{r}$, eqn. (1). The resultant radial particle flux (eqn. (2)) has been calculated for resonant modes, i.e those for which the parallel wave number $k_{\|}=\frac{m-n q}{R q}=0$. It may be written as

$$
\Gamma_{r}=-2 \frac{\lambda_{e}}{R q} \frac{v_{e, t h}}{T_{e}} D_{M}\left(P_{e}^{\prime}-e n_{e} \phi_{0}^{\prime}\right) .
$$


Here $D_{M}$ has the form of a magnetic diffusion coefficient

$$
D_{M}=R q \sum \sum \sum b_{\omega, m, n} b_{\omega, m, n}^{*} A\left(k_{\|}\right),
$$

where $b^{*}$ represents the complex conjugate and $A\left(k_{\|}\right)$is a form factor which describes the resonance width.

Using the simplified Krook model conserving only particles (as in [5]), and expanding in the parameter $\epsilon=v_{e, t h} k_{\|} / \nu<<1$, we find

$$
A^{-1}\left(k_{\|}\right)=1+\frac{v_{e, t h}^{4} k_{\|}^{4}}{\nu^{2} \omega^{2}}
$$

from which one may deduce a resonance width

$$
0 \leq\left|\frac{v_{e t h} k_{\|}}{\nu}\right| \leq\left(\frac{\omega}{\nu}\right)^{1 / 2}
$$

This value is small enough to lie within the expansion range, and is considerably smaller than that given in [4].

Evaluating the radial thermal flux

$$
Q_{r}=<b_{r} \frac{m}{2} \int\left(\left[v_{\|}-u_{\|}\right]^{2}+2 \mu B\right)\left(v_{\|}-u_{\|}\right) f d^{3} v>,
$$

we find that

$$
Q_{r}=-\frac{5}{2}\left(\frac{\lambda_{e}}{R q}\right) v_{e, t h} n_{e} D_{M} \frac{\partial T_{e}}{\partial r} .
$$

Momentum conservation in the collision term, which is neglected, for instance, in [4] and [5], yields a factor 2 in the electron particle flux and 2.5 in the electron thermal flux, but causes the ion transport to vanish, even for $k_{\|}=0$.

\section{ELECTRIC FIELD EFFECTS}

Because of the difference between the electron and ion thermal velocities, particle flow in an ergodic magnetic field is not automatically ambipolar and a radial electric field is set up to restore ambipolarity. We investigate here a model proposed by Stringer [7], in which we consider the above anomalous electron transport arising from the braided magnetic field, and neoclassical ions .

Equating the neoclassical ion flux [8] and the anomalous electron flux (eqn. 5), one finds that the radial electric field adjusts itself to the value of the poloidal rotation. For a self-consistent calculation, however, one needs two further equations. These are the equation for radial ion motion

$$
\phi_{0}^{\prime}+V_{i, \phi} B_{\theta}-V_{i, \theta} B_{\phi}=-\frac{P_{i}^{\prime}}{e n_{i}},
$$


(with $V_{i, \phi}$ the toroidal ion rotation velocity) and the toroidal momentum balance for the whole fluid

$$
m_{i} n_{i} \nu_{i} V_{i, \phi}=m_{i} \omega_{i} f_{p} \Gamma_{i}+M_{\phi}
$$

(with $\nu_{i}$ a phenomenological drag frequency to describe the momentum sink, $M_{\phi}$ a momentum source and $\left.f_{p}=B_{\theta} / B_{\phi}\right)$. The three unknowns $\phi_{0}^{\prime}, V_{i, \phi}$ and $V_{i, \theta}$ can be found from the ambipolar transport condition and these two equations.

The term with $\Gamma_{i}$ occurs in a stochastic magnetic field, as transport is not locally ambipolar and the component parallel to the field lines cannot contribute to the $\vec{j} \times \vec{B}$ forces. Thus $\Gamma_{i}$ can be replaced by the parallel electron flux $\Gamma_{e, \|}$. As the $\vec{j} \times \vec{B}$ force is a large quantity, the electric field is restricted to the value

$$
e n_{e} \phi_{0}^{\prime}-P_{e}^{\prime} \sim 0 \text {. }
$$

$M_{\phi}$ is typically too small to control the toroidal rotation, which thus follows purely from radial momentum balance (9) and neoclassical rotation (10),

$$
V_{i, \phi}=-\frac{P^{\prime}}{e n_{e} B_{\theta}}-\frac{T_{i}}{e B_{\theta}} \cdot \frac{\partial\left(\ln n_{i} T_{i}^{-\gamma}\right)}{\partial r}
$$

(where $P=P_{e}+P_{i}$ is the total pressure).

This rotation velocity can be considered an indicator of the presence of a stochastic magnetic field and reaches values large enough to be measured - for ASDEX in the $\mathrm{H}$-mode a value of about $5 \times 10^{4} \mathrm{~m} / \mathrm{s}$ may be estimated.

\section{Acknowledgements}

Valuable discussions with D.Pfirsch have led to the use of the Krook collision model. This research was supported in part by the Foundation for Research Development.

\section{References}

[1] W. Engelhardt and W.Feneberg J. Nucl. Mat. 76,77, 518 (1978)

[2] J.Neuhauser, et al. Amer. Phys. Soc. Plasma Phys. Div. meeting, Paper 5V18, San Diego (1987)

[3] A.B.Rechester, M.N.Rosenbluth Phys. Rev. Lett. 40, 38 (1978)

[4] T.Yamagishi, D.K.Bhadra Plasma Phys. \& Contr. Fus. 25, 1415 (1983)

[5] A.Thyagaraja, I.L.Robertson, F.A.Haas Plasma Phys. \& Contr. Fus. 27, 1217 (1985)

[6] P.C.Clemmow, J.P.Dougherty Electrodynamics of Particles and Plasmas AddisonWesley, London (1969)

[7] T.E.Stringer private communication

[8] L.M.Kovrizhnyk Nucl. Fus. Suppl. 12, 33 (1972) 